\title{
Aspectos Morfológicos del Arco Palmar Superficial en Individuos Adultos Mayores
}

\author{
Morphological Aspects of the Surface Palmar Arch \\ in Old Adult Individuals
}

Homero F. Bianchi ${ }^{1,2}$ \& Nicolás E. Ottone $e^{3,4,5}$

BIANCHI, H. F. \& OTTONE, N. E. Aspectos morfológicos del arco palmar superficial en individuos adultos mayores. Int. J. Morphol., 39(2):347-354, 2021.

RESUMEN: El objetivo de este trabajo consiste en el análisis de la posición del arco palmar superficial en la palma de la mano, con identificación del origen, forma de disposición, anastomosis presentes y terminación del mismo, proporcionando la experiencia propia y comparándola con los referentes del área, sin llevar adelante una comparación de clasificaciones, sino por el contrario, buscando analizar la información desde un punto de vista clínico-quirúrgico, demostrando la importancia del conocimiento de la anatomía real del arco palmar superficial al momento de abordar la anatomía vascular de la mano en todo tipo de situaciones patológicas.

PALABRAS CLAVE: Arco palmar superficial; Arteria radial; Arteria ulnar; Variaciones anatómicas; Anatomía clínica.

\section{INTRODUCCIÓN}

Continuando con la investigación de la circulación del miembro superior, seleccionamos 40 especímenes con características diferentes a las descriptas clásicamente para el arco palmar superficial (APS), que representan un $10 \%$ del total de nuestras investigaciones, siendo Arquez (2018) y Coleman \& Anson (1961), quienes señalan una mayor prevalencia de variaciones del APS. El enfoque será sobre la morfología del APS, con referencia a la posición del arco en la palma de la mano y su relación con el margen distal del retináculo flexor del carpo (RF), con el objetivo de aportar conocimientos a la anatomía vascular de la mano y su importancia en el diagnóstico y abordaje quirúrgico de la misma. No discutiremos las diferentes clasificaciones que abundan en la literatura.

\section{MATERIAL Y MÉTODO}

Se disecaron 40 miembros superiores de cadáveres adultos frescos, sin distinción de sexo, de entre 60 y 80 años de edad, inyectados con material elastomérico coloreado (lá- tex natural) por canulación de la arteria axilar. Fueron sumergidos en formaldehido al $15 \%$, y conservados en la misma solución al $10 \%$, previa inmersión en peróxido de hidrógeno al $10 \%$. Se realizó una técnica de disección habitual con instrumental estándar, de microcirugía y lupas de magnificación 2x. Las mediciones se realizaron desde el pliegue de flexión distal de la muñeca al centro del APS, y de éste al margen distal del RF.

De los 40 especímenes, en 37 se encontró APS, y en 3 especímenes existió codominancia (entre arterias radial y ulnar).

Para ubicar el APS se tomaron con referencias: el pliegue distal de la muñeca, la línea cardinal de Kaplan (Kaplan, 1961), el RF, en 33 especímenes (excepto los codominantes y los de arterias ulnares largas, 3 y 4 especímenes, respectivamente).

Definimos a los arcos palmares como Arco palmar Completo Geométrico (ACG) y Arco palmar Completo No Geométrico (ACNG), entre los cuales a su vez diferencia-

\footnotetext{
${ }^{1}$ Cirujano de Mano, Profesor Consulto, Departamento de Anatomía, Facultad de Medicina, Universidad de Buenos Aires, Buenos Aires, Argentina.

${ }^{2}$ Departamento de Anatomía, Fundación Barceló, Instituto Universitario de Ciencias de la Salud, Buenos Aires, Argentina.

${ }^{3}$ Laboratorio de Plastinación y Técnicas Anatómicas, Centro de Investigación en Ciencias Odontológicas (CICO), Facultad de Odontología, Universidad de La Frontera, Temuco, Chile.

${ }^{4}$ Departamento Odontología Integral Adultos, Facultad de Odontología, Universidad de La Frontera, Temuco, Chile.

${ }^{5}$ Centro de Excelencia en Estudios Morfológicos y Quirúrgicos (CEMyQ), Facultad de Medicina, Universidad de La Frontera, Temuco, Chile.
} 
mos por la presencia de sinuosidades en el ACNG que le provocan la pérdida de la geometría característica del arco. A su vez, identificamos un Arco palmar Completo Ulnar No Geométrico (ACUNG), en el cual predomina la arteria ulnar, con pérdida de geometría del arco también por presencia de sinuosidades.

La totalidad de acrónimos utilizados en este trabajo son los siguientes: A1EIO; A2EIO, A3EIO, A4EIO: arteria segundo, tercer y cuarto espacio interóseo; ADPPRP: arteria digital palmar propia radial del pulgar; ADPPUP: arteria digital palmar propia ulnar del pulgar; ADPC: arterias digitales palmares comunes; ACG: arco completo geométrico; ACNG: arco completo no geométrico; ACUNG: arco completo ulnar no geométrico; AM: arteria mediana; ADPPM5D: arteria digital palmar propia medial del $5^{\circ}$ dedo; AR: arteria radial; ARI: arteria digital palmar propia radial del índice; ARS: arteria radial superficial; AU: arteria ulnar; AUS: arteria ulnar superficial; EIO: espacio interóseo; AMTC: arteria metacarpiana; NM: nervio mediano; UN: nervio ulnar; TF tendones de los músculosflexores.

\section{RESULTADOS Y DISCUSIÓN}

Lo primero a señalar es que todos los arcos palmares, excepto los ACG, mostraron flexuosidades, lo cual los aleja de los diferentes esquemas que se aprecian en las referencias de diferentes publicaciones, pero se reconocen como las sinuosidades mencionadas por Chambardel, y observadas en las preparaciones de Ottone et al. (2010), Tagil et al. (2007) y en las figuras de Janevski (1982) y Kaplanoglu \& Beton (2017), habituales en la edad avanzada.

La clasificación de los hallazgos siguió lo establecido en las referencias, en relación con las anastomosis arteriales (Dubreuil-Chambardel, 1926; Adachi, 1928; Coleman \& Anson; Janevski; Al-Turk \& Metcalf, 1984; Tagil et al.; Ottone et al.; Aughsteen et al., 2012; Gharravi et al., 2013; Saxena et al., 2013; Joshi et al., 2014; Mbaka et al., 2014; Tasom et al., 2014; Hashem et al., 2018). De los hallazgos resulta que los APS completos son mayoría, aunque en nuestro caso la prevalencia es del tipo ACUNG (40\%) (Libersa et al., 1982). El tipo ACG resulta en menor número que el tipo ACNG (Figs. 1A,B).

Los arcos incompletos, resultan de la ausencia de anastomosis arteriales con ramas de la arteria radial, codominantes o ausencia de la A1EIO (Figs. 1C,D). Dos casos figuran con un signo de interrogación, porque si bien son del tipo AR/AU (Ottone et al.), muestran una anastomosis que las une, por lo cual, si por anastomosis se trata entre los sistemas radial ulnar ¿deberían definirse como ACNG intermedios? (Fig. 1E). No se ha encontrado en las referencias una disposición similar.

De los 9 ACNG, 6 fueron considerados irregulares. De estos 6 casos:

- En dos casos, uno presentó un APS corto, y el otro un APS muy irregular, con variación de origen de la ADPPM5D con doble anastomosis con la arteria radial superficial (ARS) y A1EIO (Figs. 1F y 2A);

- En dos casos fueron similares, con un trazado del APS irregular por sobre los TF. La AUS emite un tronco que da la A4EIO y la A3EIO, naciendo de esta, la A2EIO, origen a su vez, de la (A1EIO), que se une a la A1EIOD y a la ARS (pretenar) en una doble anastomosis, originando la AUS, la ADPPM5D (Figs. 2B,C);

- En dos casos fueron incluidos solo por el tipo de anastomosis, con predominio de la AUS (Figs. 2D,E): ambos muestran anastomosis de la AU con el sistema radial, uno de ellos doble como en los casos precedentes, pero por la morfología de ambos, concluimos que no deberían ser considerados APS. El total de doble anastomosis, incluyendo a la ARS, suma seis especímenes.

Todos los casos de ACUNG mostraron, además de las sinuosidades, dos trayectos: ascendente, $40 \%$ (Fig. 1F) y transverso, 22,5 \% (Fig. 1C). Los ACG y ACNG regulares fueron excluidos, junto con los AR/AU y los dos casos de ACNG con predominio de la AUS (Figs. 2D,E), por su irregularidad. Cinco de los ACUNG con trayecto ascendente, mostraron una terminación corta transversal (Fig. 1D). Es posible (y discutible) que a partir del punto en que la AUS se curva hacia lateral, las flexuosidades influyan en la dirección del arco. Un caso de irregularidad extrema se incluyó entre los transversos (Fig. 1F).

La extensión del APS, difícil de cuantificar a causa de las flexuosidades, se consideró tomando como puntos de referencia, para los ACNG irregulares y los ACUNG, los TF de los dedos $3^{\circ}$ y $2^{\circ}$, y los EIO $3^{\circ}$ y $2^{\circ}$. Con mayor frecuencia, $37,5 \%$ de estos arcos se extienden hasta el tendón flexor del tercer dedo (Figs. 1F, 2B, 3A); en segundo lugar, estos arcos se extienden hasta el 2EIO, correspondiendo a un 22,5\% de los casos (Fig. 1C), considerado por Ottone et al. como habitual, de lo cual resulta, que la suma de los APS terminados en el 3EIO (Figs. 3D y 4A) y el 4TF, muestra APS más cortos en un número mayor que lo que suele verse en las ilustraciones de las referencias (Coleman \& Anson; Al-Turk \& Metcalf; Ruengsakulrach et al., 2001; Tagil et al.; Ottone et al.; Joshi et al.; Ghrravi). El acortamiento del APS puede verse en los trabajos de Kaplanoglu $\&$ Beton, y es mencionado por Browning \& Morton (1955). 

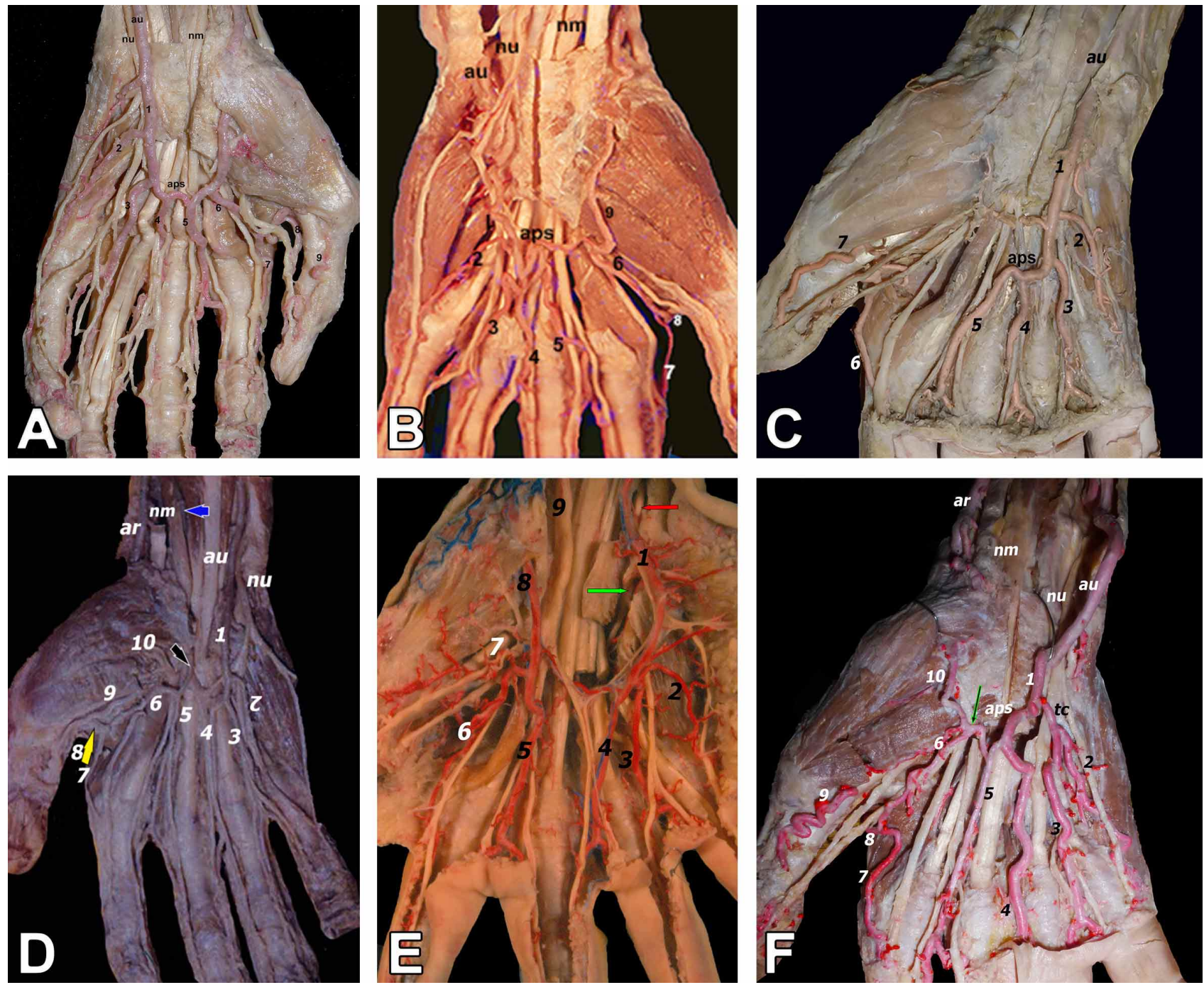

Fig. 1. Disposiciones del arco palmar superficial. A y B. Arco Palmar Completo Geométrico y Arco Palmar Completo No Geométrico. au, arteria ulnar; un, nervio ulnar; nm, nervio mediano; aps, arco palmar superficial; 1 , arteria ulnar superficial; 2, arteria digital palmar propia medial del $5^{\circ}$ dedo; 3 , arteria del cuarto espacio interóseo; 4, arteria del tercer espacio interóseo; 5 , arteria del segundo espacio interóseo; 6, arteria del primer espacio interóseo; 7, arteria digital palmar propia radial del índice; 8, arteria digital palmar propia ulnar del pulgar; 9, arteria digital palmar propia radial del pulgar. C. Arco Palmar Superficial Incompleto. au, aps, 2, 4, 5 (igual que A y B); 6, arteria digital palmar propia radial del índice; 7, arteria digital palmar propia radial del pulgar. D. Arco Palmar Superficial Incompleto. un, au, nm, 1, 2, 3, 4, 5, 6, 8, 9 (igual que A y B); ar, arteria radial; 7 arteria digital palmar propia radial del índice anastomosada con la primera arteria metacarpiana (flecha amarilla); 10, rama tenar; flechas negra y azul, arteria mediana. E. Arco Palmar Completo No Geométrico Intermedio. 1, 2, 3, 4, 5 (igual que A y B); 6, arteria digital palmar propia radial del índice; 7, arteria del primer espacio interóseo; 8, arteria radial superficial; Flecha roja, nervio ulnar; Flecha verde, ramo profundo del nervio ulnar. F. Arco Palmar Completo No Geométrico Irregular. au, nu, nm, ar, aps, 1, 2, 3, 4, 5, 6, 7, 8, 9 (igual que A y B); tc, tronco común entre arteria digital palmar propia medial del $5^{\circ}$ dedo y arteria del cuarto espacio interóseo; 10 , arteria radial superficial; flecha verde, doble anastomosis entre arteria del segundo espacio interóseo, arteria radial superficial y arteria del primer espacio interóseo.

En la serie, las ADPC muestran flexuosidades acompañando a las del APS, impidiendo que las mismas alcancen en forma directa los EIO, obligando a las arterias a "saltar" sobre los tendones para alcanzar su posición. En ocasiones, las sinuosidades se observan en las ADPC ya ubicadas en el EIO, sobrepasando al tendón por tramos.
(Figs. 1A,B,C, 2A y 3C). Esto es frecuente en la A2EIO, cuando nace sobre el TF del tercer dedo o con menor frecuencia en el 3EIO. El APS, en siete casos, se divide en dos ramas, medial y lateral, de longitud variable. La rama lateral da origen, por separado, a las ADPC de los EIO $2^{\circ} \mathrm{y}$ $3^{\circ}$ (Figs. 3A,D,E), mientras que el origen común de las 

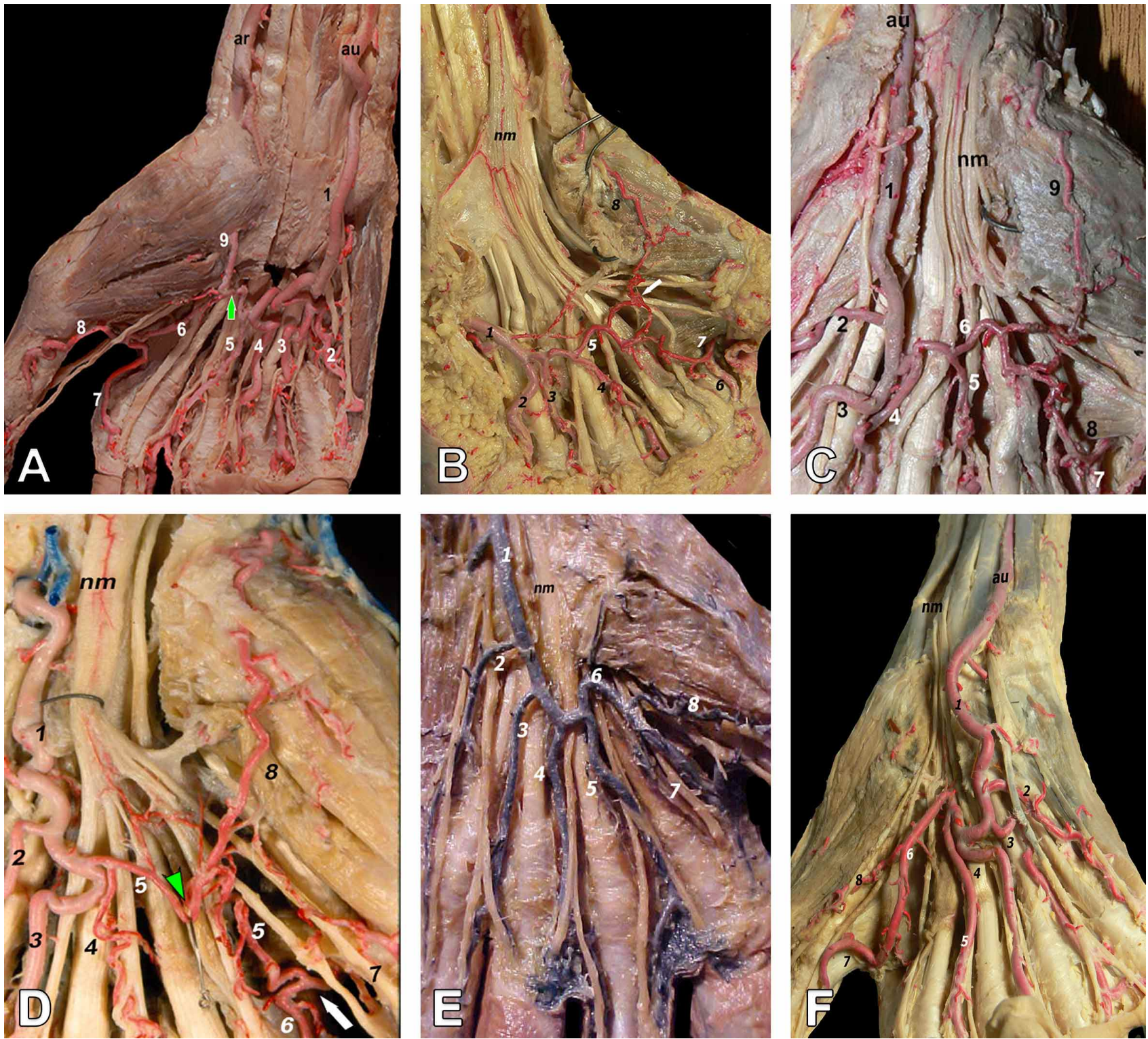

Fig. 2. A. Arco Palmar Completo No Geométrico Irregular. au, arteria ulnar; ra, arteria radial; 1, arteria ulnar superficial; 2, arteria digital palmar propia medial del $5^{\circ}$ dedo; 3 , arteria del cuarto espacio interóseo; 4 , arteria del tercer espacio interóseo; 5 , arteria del segundo espacio interóseo; 6 , arteria del primer espacio interóseo; 7, arteria digital palmar propia radial del índice; 8, arteria digital palmar propia ulnar del pulgar; 9, arteria radial superficial; flecha verde, anastomosis entre arteria radial superficial y arteria del primer espacio interóseo dorsal. B. Arco Palmar Completo No Geométrico Irregular, sobre los tendones flexores. A. mn, nervio mediano; 1, arteria ulnar superficial; 2, arteria del cuarto espacio interóseo; 3, arteria del tercer espacio interóseo; 4, arteria del segundo espacio interóseo; 5 , arteria del primer espacio interóseo; 6, arteria digital palmar propia radial del índice; 7, anastomosis entre arteria digital palmar propia radial del índice y arteria del primer espacio interóseo; 8 , arteria radial superficial; flecha blanca, anastomosis entre arteria del primer espacio interóseo y arteria radial superficial. C. Arco Palmar Completo No Geométrico Irregular, sobre los tendones flexores. ua, arteria ulnar; 1, 2, 3, 4, 5, 6 (igual que B); 7, arteria digital palmar propia radial del índice; 8, arteria del primer espacio interóseo dorsal; 9, arteria radial superficial; flecha blanca, anastomosis entre arteria del primer espacio interóseo (6) y arteria radial superficial (9). D. Arco Palmar Completo No Geométrico Irregular, con predominio de la arteria ulnar superficial. mn, 1, 2, 3, 4, 5, 6, 8 (igual que B); 7, arteria digital palmar propia ulnar del pulgar; flecha verde, anastomosis entre arteria del primer espacio interóseo (5) y arteria radial superficial (8); flecha blanca, arteria del primer espacio interóseo dorsal. E. Arco Palmar Completo No Geométrico Irregular, con predominio de la arteria ulnar superficial. mn, 2, 3, 4, 5, 6, 7, 8, (igual que A); 1, arteria ulnar. F. 1, 2, 3, 4, 5, 6 (igual que A); ua, arteria ulnar; 7, arteria digital palmar propia ulnar del pulgar; 8, arteria digital palmar propia radial del pulgar. 

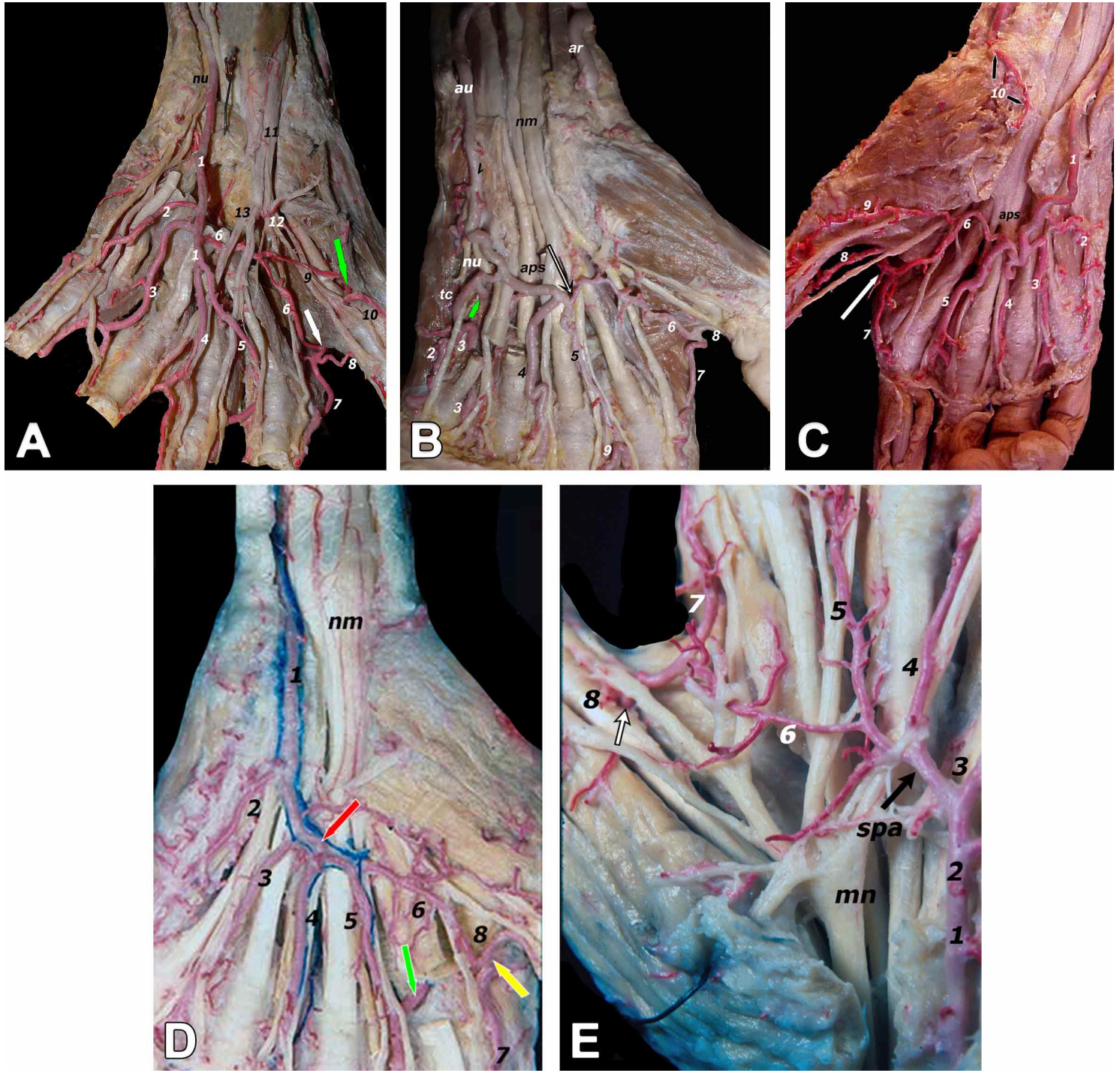

Fig. 3. A. Predominio de arteria ulnar superficial larga en la corformación del arco palmar superficial. un, nervio ulnar; 1, arteria ulnar superficial; 2, arteria digital palmar propia medial del $5^{\circ}$ dedo; 3 , arteria del cuarto espacio interóseo; 4, arteria del tercer espacio interóseo; 5, arteria del segundo espacio interóseo; 6, arteria del primer espacio interóseo; flecha blanca, anastomosis entre arteria del primer espacio interóseo (6) y arteria del primer espacio interóseo dorsal; 7, arteria digital palmar propia radial del índice; 8, arteria digital palmar propia ulnar del pulgar; 9, rama de arteria del primer espacio interóseo (6) anastomosada con arteria digital palmar propia radial del pulgar (10); 10, arteria digital palmar propia radial del pulgar (procedente de la primera arteria metacarpiana (flecha verde); 11, 12, 13, nervio mediano. B. Predominio de arteria ulnar superficial larga en la corformación del arco palmar superficial, presentando como rama terminal la A2EIO (arteria del segundo espacio interóseo). 1, 2, 3, 4, 5, 6, 7, 8 (igual que A); ar, arteria radial; au, arteria ulnar; nu, nervio ulnar; aps, arco palmar superficial; tc, tronco común entre 2 y 3; 9, rama anastomótica entre arteria del segundo espacio interóseo dorsal y arteria del segundo espacio interóseo; flecha verde, arteria ulnar profunda; flecha blanca, origen de arterias del primer y segundo espacio interóseo. C. Predominio de arteria ulnar superficial larga en la corformación del arco palmar superficial, proporcionando la arteria del primer espacio interóseo, como rama terminal. 1, 2, 3, 4, 5, 6, 7, 8, (igual que A); aps, arco palmar superficial; 9, arteria digital palmar propia radial del pulgar; 10, rama lateral de la arteria radial para el pulgar; flecha blanca, arteria del primer espacio interóseo. D. Predominio de arteria ulnar superficial larga en la corformación del arco palmar superficial, finalizando el arco en el 3EIO (tercer espacio interóseo). mn, nervio mediano; 1, 2, 3, 4, 5, 6, 7, 8 (igual que A); flecha negra, division de la arteria ulnar superficial en arterias del tercer y segundo espacio interóseo; flecha amarilla, anastomosis con arteria del primer espacio interóseo dorsal; flecha verde, segunda arteria metacarpiana. E. Predominio de arteria ulnar superficial en la corformación del arco palmar superficial. mn, 1, 2, 3, 4, 5, 6, 7, 8 (igual que D); flecha negra, arco palmar superficial proporcionando las arterias del segundo y tercer espacio interóseo; flecha blanca, primera arteria metacarpiana proporcionando las arterias radial del índice (7) y arteria digital palmar propia ulnar del pulgar (8), anastomosada con la arteria del primer espacio interóseo (6). 
ADPC de los EIO $3^{\circ}$ y $4^{\circ}$ se observó en dos de los especímenes clasificados como ACUNG irregulares (Figs. 2B,C). Hallazgos similares han sido reportados por Onderoglu et al. (1997), Bataineh \& Moqattash (2006), Blajoka, Ottone et al. y Mbaka et al.

La A1EIO, cuarta ADPC cuando está presente, (Coleman \& Anson; Al-Turk \& Metcalf; Mbaka et al.; Hashem et al.), ha sido considerada desde Tandler (Coleman \& Anson), rama terminal del APS. Nuestros resultados muestran más de un origen de la A1EIO, siendo el más frecuente el APS (Figs. 2F, 3C,D,E y 4B), seguido por la A2EIO (Figs. 1F y 3B) y la ARS (Figs. 1A,B,E). Un caso infrecuente fue el origen común de la A1EIO con la A2EIO, ambas nacidas de la A3EIO (Fig. 4A). Onderoglu et al. mencionan el origen común de estas arterias, pero del APS. En tres casos, si bien la terminación es la A1EIO, sería posible interpretar un origen casi equidistante de la A1EIO y la A2EIO (Figs. 2E y 4B). Reconocemos que no siempre es fácil determinar cuando la A2EIO es colateral o arteria terminal, por lo difícil de establecer un límite entre esta y el APS (Figs. 3C y 4C). Chambardel cita a Poirier, quien consideró que el APS parecía continuarse con la A2EIO. Singh lo menciona para la A2EIO y la A3EIO. La A2EIO puede considerarse terminal cuando es la última arteria y no existe la A1EIO, como en los tres casos de arcos incompletos (Fig. 1C), similar al caso de la Figura 1E de la comunicación de Libersa et al.

La terminación de la A1EIO fue por división en arteria digital palmar propia radial del índice (ARI) y arteria digital palmar propia ulnar del pulgar (ADPPUP) (TandlerPoirier citados por Chambardel), en mayor proporción la primera (Figs. 3A,C y 4A), o por anastomosis con la 1AIOD, algo menor (Figs. 3D,E). Cinco casos mostraron una doble anastomosis entre la A1IOD y la ARS. (Figs. 1F y 2A,B,C). En un caso, la A2EIO se une con la A2IOD, evento reportado previamente (Bianchi et al., 2018) (Fig. 1F).

Diferentes autores realizaron mediciones para ubicar APS en relación con los pliegues palmares. Testut \& Jacob (1961) ubicaron el APS $17 \mathrm{~mm}$ distal al pliegue palmar medio, mientras que, en relación al pliegue distal de la muñeca, lo ubica a $45 \mathrm{~mm}$. Tomando de referencia este pliegue, McLean et al. (2008) sitúa al APS a 51,8 mm +/7,56 , siendo para nosotros la distancia, en promedio, de 45 $\mathrm{mm}$, igual al de Testut \& Jacob. Al sumar los valores de los APS ascendentes y transversos, obtuvimos con sorpresa, el mismo valor en la sumatoria: $45 \mathrm{~mm}$, pese a las diferencias observadas en los casos individuales. Los ACG y ACNG mostraron una distancia de $55 \mathrm{~mm}$, cercana a los valores de McLean et al. creemos por la diferente geometría observada.
Por su morfología destacamos aquellos con AUS largas, ubicados entre 100 y $75 \mathrm{~mm}$ con un promedio de 83,75 (Figs. 2B,C,D y 3A), lo cual, en casos de incisiones distales, expone a lesiones de la arteria o sus ramas, al igual que en incisiones paralelas o sobre la misma. En relación a la línea cardinal de Kaplan, Panchal \& Trzeciak (2020) ubican al APS 6 mm distal a la misma, McLean et al. lo hace a $15+/-8,60 \mathrm{~mm}$. En nuestro trabajo, en 19 preparaciones, el APS se encuentra a $10 \mathrm{~mm}$ ( 2 a $15 \mathrm{~mm}$ ) distal de la línea, mientras que, en 14 casos, se ubicó proximal o a nivel de la misma. Creemos que estas variaciones de longitud se deben a las modificaciones morfológicas del APS. La distancia al margen distal del RF guarda importancia en relación con las lesiones vasculares reportadas durante la liberación del túnel carpiano, según lo reportado por Bilgin et al. (2004) quienes reportaron distancias de 2 a $22 \mathrm{~mm}$. Nuestra serie mostro una media de $15 \mathrm{~mm}$ (2-25 mm), coincidente con valores reportados (Figs. 1E y 2A). Hay que considerar que una AUS puede ubicarse cercana a la línea media (Mbaka et al.) y verse comprometida en los abordajes de la región (Fig. 4B).

Si bien el número de preparaciones no se consideren numerosas, deberìan ser tenidas en mente al abordar manos de pacientes adultos mayores en especial, ya que pueden sorprender al cirujano al momento de la cirugía. Las flexuosidades pueden modificar la morfología del APS, su longitud, su relación con puntos de reparo, y la relación con el margen distal del RF, a lo cual se suman las variaciones de las ADPC previo a, y dentro de los EIO, su relación con los tendones flexores y los nervios palmares digitales comunes a causa de las sinuosidades mencionadas, y las modificaciones en su longitud. Conocer estas variaciones ayudaran en la interpretación de angiografías y ante intervenciones que se deban realizar en abordajes palmares tanto reconstructivas como plásticas, en especial la retracción de Dupuytren, sumadas a posible patología vascular. La relación con el margen distal del RF ha sido mencionada en varias referencias en relación a los daños del APS en cirugías de liberación del nervio mediano, ya sea en técnica a cielo abierto o cerrado y deberá tenerse en cuenta para evitar daños al APS (MacDonald et al., 1978; Murphy Jr. et al., 1994; Shinya et al., 1995; Bilgin et al.; Sacks et al., 2007; Didailler et al., 2011). La posición del APS en relación con el pliegue cutáneo proximal o con la línea cardinal de Kaplan o la muy similar línea de Boeckel (Delorme, 1882; Testut \& Jacob), con distancia entre $1 \mathrm{a} 10 \mathrm{~mm}$ distal a la misma, son puntos de reparo importantes para su localización y si bien el promedio acuerda con resultados previos oscila entre valores algo extremos al incluir a las AUS largas. 

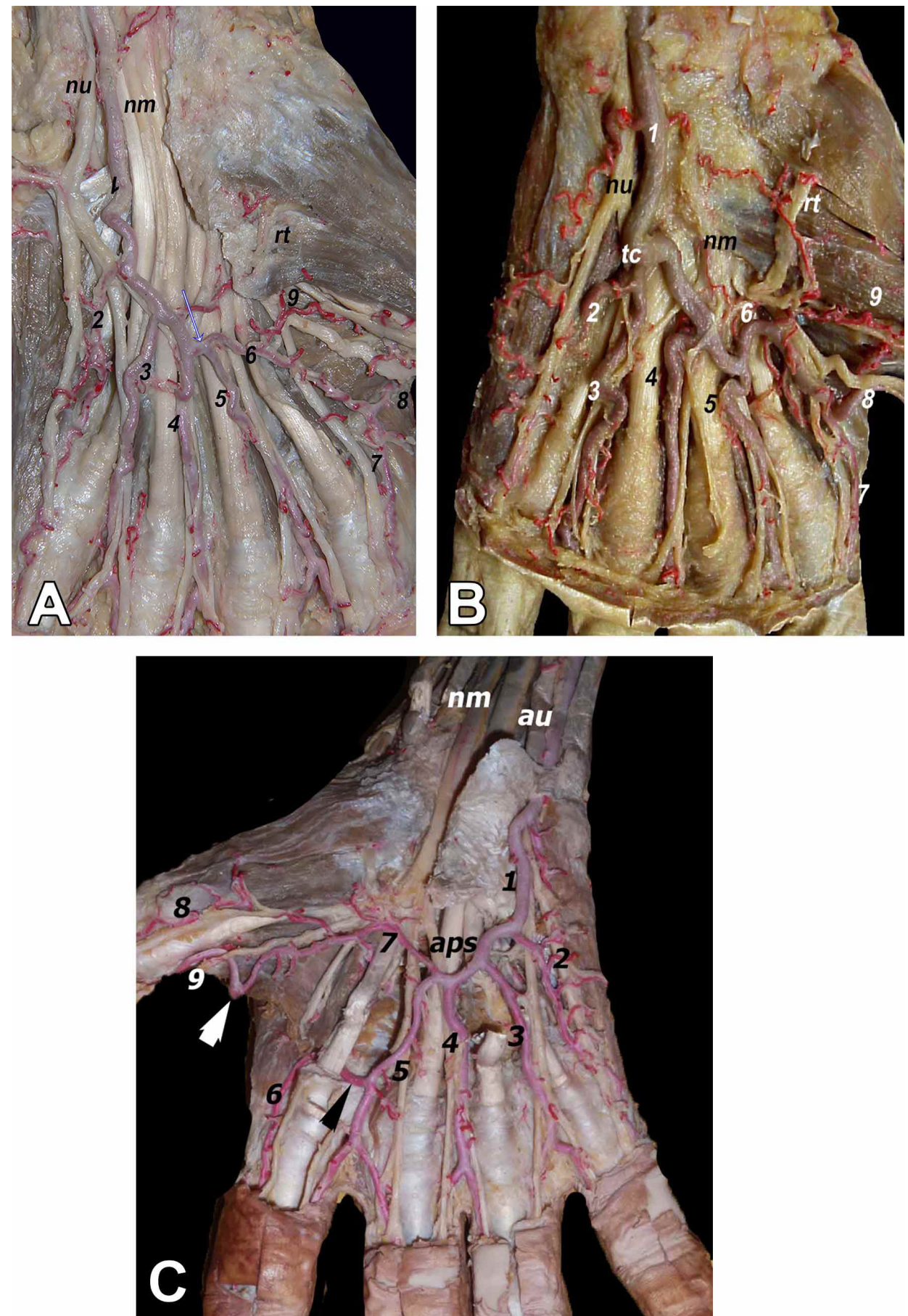

Fig. 4. A. Arco palmar Completo Ulnar No Geométrico (ACUNG), que presenta el origen común de la A1EIO (6) con la A2EIO (5), ambas nacidas de la A3EIO (4). 1, arteria ulnar superficial; 2, arteria digital palmar propia medial del $5^{\circ}$ dedo; 3 , arteria del cuarto espacio interóseo; 4, arteria del tercer espacio interóseo; 5, arteria del segundo espacio interóseo; 6, arteria del primer espacio interóseo; flecha blanca, origen común de las arterias del primer y segundo espacio interóseo desde la arteria del tercer espacio interóseo; 7, arteria digital palmar propia radial del índice; 8, arteria digital palmar propia ulnar del pulgar; 9, rama lateral del pulgar de la arteria radial. B. Arco palmar Completo Ulnar No Geométrico (ACUNG). nm, nervio mediano; nu, nervio ulnar; rt, rama tenar; tc, tronco común entre 2 y 3; 1, 2, 3, 4, 5, 6, 7, 8 (igual que A); 9, arteria digital palmar propia radial del pulgar. C. Arco palmar Completo Ulnar No Geométrico (ACUNG). au, arteria ulnar; nm, nervio mediano; 1, 2, 3, 4, 5 (igual que A) ; 6, arteria digital palmar propia radial del índice; 7, arteria del primer espacio interóseo; 8, arteria digital palmar propia radial del pulgar; 9, arteria digital palmar propia ulnar del pulgar; flecha blanca, anastomosis entre arteria del primer espacio interóseo y arteria del primer espacio interóseo dorsal; flecha negra, primera arteria metacarpiana. 


\section{CONCLUSIONES}

La presente serie, si bien no muy numerosa, analiza aspectos no profundizados de la morfología del APS, que no por ser habituales, no deben dejar de ser tomadas en cuenta, en el abordaje de la palma de la mano de pacientes añosos, pudiendo las estructuras estar en peligro de ser dañadas por la alteración de posición y relación de las mismas, además de contribuir al conocimiento anatómico detallado de la circulación superficial de la mano e interpretación de imágenes angiográficas. Creemos que hay motivo para considerar que el término arco debería reservarse para los ACG, y el de AUS para el resto.

BIANCHI, H. F. \& OTTONE, N. E. Morphological aspects of the surface palmar arch in old adult individuals. Int. J. Morphol., 39(2):347$354,2021$.

SUMMARY: The objective of this work consists in the analysis of the position of the superficial palmar arch in the palm of the hand, with identification of the origin, form of disposition, present anastomosis and termination of the same, providing the own experience and comparing it with the referents of the area, without carrying out a comparison of classifications, but on the contrary, seeking to analyze the information from a clinical-surgical point of view, demonstrating the importance of knowledge of the real anatomy of the superficial palmar arch when addressing the vascular anatomy of the hand in all kinds of pathological situations.

KEY WORDS: Superficial palmar arch; Radial artery; Ulnar artery; Anatomical variations; Clinical anatomy.

\section{REFERENCIAS BIBLIOGRÁFICAS}

Adachi, B. Das Arteriensystem der Japaner. Kyoto, Marusen Co., 1928.

Al-Turk, M. \& Metcalf, W. K. A study of the superficial palmar arteries using the Doppler Ultrasonic Flowmeter. J. Anat., 138(Pt. 1):27-32, 1984.

Arquez, H. Arare finding in the hand irrigation. Bangladesh J. Med. Sci., 17(3):496$500,2018$.

Aughsteen, A. A. Case report of a new variant of double incomplete superficial palmar arch. Anat. Sci. Int., 87(1):56-9, 2012.

Bataineh, Z. M. \& Moqattash, S. T. A complex variation in the superficial palmar arch. Folia Morphol. (Warsz.), 65(4):406-9, 2006.

Bianchi, H. F.; Vargas, C. A. \& Ottone, N. E. Unusual ending of the second dorsal metacarpal artery as proper palmar digital arteries. Folia Morphol. (Warsz.), 77(2):393-6, 2018.

Bilgin, S. S.; Kose, K. C.; Demirtas, A. M. \& Adiyaman, S. Necessity of inspection of superficial palmar arch by blunt dissection from exit portal in endoscopic carpal tunnel release. Plast. Reconst. Surg., 114(2):602-3, 2004.

Browning, H. C. \& Morton, D. E. The arterial pattern in the hand. Yale J. Biol. Med., 28(1):37-55, 1955.

Coleman, S. S. \& Anson, B. J. Arterial patterns in the hand based upon a study of 650 specimens. Surg. Gynecol. Obstet., 113:409-24, 1961

Delorme, E. De la Ligature des artères de la paume de la main et des artères de la plante du pied. Paris, Masson, 1882.

Didailler, P.; Ragois, P. \& Morales, P. Pseudo-anévrisme de l'arcade palmaire superficielle après chirurgie endoscopique du canal carpien. À propos d'une observation. Chir. Main, 30(1):73-5, 2011.

Dubreuil-Chambardel, L. Variations des artères du Membre Supérieur. Paris, Masson, 1926.
Gharravi, A. M.; Azandeh, S.; Gholami, M. R. \& Nejad, D. B. The incomplete superficial palmar arch. J. Orthop. Case Rep., 3(2):32-4, 2013.

Hashem, A. M.; Knackstedt, R. W.; Bernard, S.; Hendrickson, M.; McBride, J. M. $\&$ Djohan, R. Variations in the origins and absence of the common digital arteries of the hand: a cadaveric study. J. Hand Surg. Eur. Vol., 43(10):1054-8, 2018.

Janevski, B. K. Angiography of the Upper Extremity. Londres, Springer, 1982.

Joshi, S. B.; Vatsalaswamy, P. \& Bahetee, B. H. Variation in formation of superficial palmar arches with clinical implications. J. Clin. Diagn. Res., 8(4):AC069, 2014

Kaplan, E. B. Anatomía Funcional y Quirúrgica de la Mano. Aires, Artecnica, 1961.

Kaplanoglu, H. \& Beton, O. Evaluation of anatomy and variations of superficial palmar arch and upper extremity arteries with CT angiography. Surg. Radiol. Anat., 39(4):419-26, 2017.

Libersa, C.; Francke, J. P.; Mauppin, J. M.; Bailleul, J. P. \& Gamblin, P. The arterial supply to the palm of the hand (arteriae palmae manus). Anat. Clin., 4:33-45, 1982.

MacDonald, R. I.; Lichtman, D. M.; Hanlon, J. J. \& Wilson, J. N. Complications of surgical release for carpal tunnel syndrome. J. Hand Surg. Am., 3(1):70-6, 1978.

Mbaka, G.; Ejiwunmi, A. B. \& Olabiyi, O. Pattern of variations in superficial palmar arch in 134 Negro cadaveric hands. Ital. J. Anat. Embryol., 119(3):153-62, 2014.

McLean, K. M.; Sacks, J. M.; Kuo, Y. R.; Wollstein, R.; Rubin, J. P. \& Lee, W. P. A. Anatomical landmarks to the superficial and deep palmar arches. Plast. Reconstr. Surg., 121(1):181-5, 2008

Murphy Jr., R. X.; Jennings, J. F. \& Wukich, D. K. Major neurovascular complications of endoscopic carpal tunnel release. J. Hand Surg. Am., 19(1):1148, 1994.

Onderoglu, S.; Basar, R.; Erbil, K. M. \& Cumhur, M. Complex variation of the superficial palmar arch--case report. Surg. Radiol. Anat., 19(2):123-5, 1997.

Ottone, N. E.; Prum, N.; Dominguez, M.; Blasi, E.; Medan, C.; Shinzato, S.; Finkelstein, D. \& Bertone, V. H. Analysis and clinical importance of superficial arterial palmar irrigation and its variants over 86 cases. Int. J. Morphol. 28(1):157-64, 2010

Panchal, A. P. \& Trzeciak, M. A. The clinical application of Kaplan's cardinal line as a surface marker for the superficial palmar arch. Hand (N. Y.), 5(2):155-99, 2010.

Ruengsakulrach, P.; Eizenberg, N.; Fahrer, C.; Fahrer, M. \& Buxton, B. F. Surgical implications of variations in hand collateral circulation: anatomy revisited. J. Thorac. Cardiovasc. Surg., 122(4):682-6, 2001.

Sacks, J. M.; Kuo, Y. R.; Mclean, K.; Wollstein, R. \& Lee, W. P. A. Anatomical relationships among the median nerve thenar branch, superficial palmar arch, and transverse carpal ligament. Plast. Reconstr. Surg., 120(3):713-8, 2007.

Saxena, A.; Agarwal, K. K.; Ray, B. \& Pyrtuh, S. A rare finding of the superficial palmar arch-developmental and clinical significance. J. Clin. Diagn. Res., 7(4):706-8, 2013

Shinya, K.; Lanzetta, M. \& Conolly, W. B. Risk and complications in endoscopic carpal tunnel release. J. Hand Surg. Br., 20(2):222-7, 1995.

Tagil, S. M.; Aynur, E. C.; Tunç, C. O.; Mustafa, B. \& Ahmet, S. Variations clinical importance of the superficial palmar archs. D. Ü. T\$p. Fak. Derg., 14(2):11-6, 2007.

Tasom, A.; Sinthubua, A.; Sananpanich, K. \& Mahakkanukrauh, P. Cadaveric study of superficial palmar arch variations in northern Thai population by using epoxy resin injection technique. J. Med. Assoc. Thai., 97(12):1352-7, 2014

Testut, L. \& Jacob, O. Tratado de Anatomía Topográfica con Aplicaciones

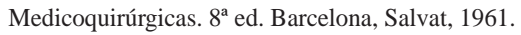

Dirección para correspondencia:

Dr. Nicolás E. Ottone

Profesor Asociado

Laboratorio de Plastinación y Técnicas Anatómicas

Centro de Investigación en Ciencias Odontológicas (ClCO)

Facultad de Odontología

Universidad de La Frontera

Temuco, CHILE

Recibido: 08-10-2020

Accepted:14-12-2020

E-mail: nicolas.ottone@ufrontera.cl

Sitio web: www.plastinacion.com 